\title{
Undirected Graphs of Entanglement 2
}

\author{
Walid Belkhir and Luigi Santocanale \\ Laboratoire d'Informatique Fondamentale de Marseille \\ Université de Provence
}

\begin{abstract}
Entanglement is a complexity measure of directed graphs that origins in fixed point theory. This measure has shown its use in designing efficient algorithms to verify logical properties of transition systems. We are interested in the problem of deciding whether a graph has entanglement at most $k$. As this measure is defined by means of games, game theoretic ideas naturally lead to design polynomial algorithms that, for fixed $k$, decide the problem. Known characterizations of directed graphs of entanglement at most 1 lead, for $k=1$, to design even faster algorithms. In this paper we give two distinct characterizations of undirected graphs of entanglement at most 2. With these characterizations at hand, we present a linear time algorithm to decide whether an undirected graph has this property.
\end{abstract}

\section{Introduction}

Entanglement is a complexity measure of finite directed graphs introduced in $[1,2]$ as a tool to analyze the descriptive complexity of the Propositional Modal $\mu$-calculus. Roughly speaking, its purpose is to quantify to what extent cycles are intertwined in a directed graph. Its game theoretic definition - by means of robbers and cops - makes it reasonable to consider entanglement a generalization of the tree-width of undirected graphs [3] to another kind of graphs, a role shared with other complexity measures appeared in the literature [4-7].

A peculiar aspect of entanglement, and also our motivation for studying it among the other measures, is its direct filiation from fixed point theory. Its first occurrence takes place within the investigation of the variable hierarchy $[8,9]$ of the Propositional Modal $\mu$-Calculus [10]. The latter, hereby noted $\mathbb{L}_{\mu}$, is nowadays a well known and appreciated logic, capable to express many computational properties of transition systems while allowing their verification in some feasible way. As a $\mu$-calculus [11] $\mathbb{L}_{\mu}$ increases the expressive power of Hennessy-Milner logic, i.e. multimodal logic $\mathbb{K}$, by adding to it least and greatest fixed point operators that bind monadic variables. Showing that there are $\mu$-formulas $\phi_{n}$ that are semantically equivalent to no formula with less than $n$ bound variables is the variable hierarchy problem for a $\mu$-calculus. Such a hierarchy is also meaningful in the simpler setting of iteration theories [12].

The relationship between entanglement and the number of bound variables in a $\mu$-term might be too technical to be elucidated here. Let us say, however, that entanglement roughly is a syntactic analogous of the variable hierarchy, the 
latter being defined only w.r.t. a given semantics. To argue in this direction, the relevant fact is Proposition 14 of [1], stating that the entanglement of a directed graph is the minimal feedback of its finite unravellings.

A second important topic in fixed point theory is the model checking problem for $\mathbb{L}_{\mu}$. The main achievement of [1] states that parity games whose underlying graphs have bounded entanglement can be solved in polynomial time. This is a relevant result for the matter of verification, since model checking $\mathbb{L}_{\mu}$ is reducible in linear time to the problem of deciding the winner of a parity game. Berwanger's result calls for the problem of deciding whether a graph has entanglement at most $k$, a problem which we address in this paper. When settled, we can try to exploit the main result of [1], for example by designing algorithms to model check $\mathbb{L}_{\mu}$ that may perform well in practice. We shall argue that, for fixed $k$, deciding whether a graph has entanglement at most $k$ is a problem in the class $\mathrm{P}$. The algorithms solving these problems can be combined to show that deciding the entanglement of a graph is in the class EXPTIME. We have no reasons to believe that the problem is in NP. Let us mention on the way that a problem that we indirectly address is that of solving parity games on undirected graphs. These games can be solved in linear time if Eva's and Adam's moves alternate. Yet, the complexity of the problem is not known if consecutive moves of the same player are allowed.

In this paper we show that deciding whether an undirected graph $G$ belongs to $\mathcal{U}_{2}$, the class of undirected graphs of entanglement at most 2 , can be solved in time $O\left(\left|V_{G}\right|\right)$. We shall present an algorithm that crucially depends on two characterizations of the class $\mathcal{U}_{2}$. One of them proceeds by forbidden subgraphs: an undirected graph belongs to $\mathcal{U}_{2}$ if and only if it does not contain (i) a simple cycle of length strictly greater than 4 , (ii) a length 3 simple cycle whose vertices have all degree 3 , (iii) a length 4 simple cycle with two adjacent vertices of degree 3. A second characterization constructs the class $\mathcal{U}_{2}$ from a class of atomic graphs, called the molecules, and an operation, the legal collapse, that glues together two graphs along a prescribed pair of vertices.

The two characterizations may be appreciated on their own, independently of the algorithm they give rise. Entanglement is an intrinsically dynamic concept, due to its game theoretic definition. As such it is not an easy object of study, while the two characterizations prepare it for future investigations with standard mathematical tools. They also suggest that entanglement is a quite robust notion, henceforth worth being studied independently of its fix-point theoretic background. As a matter of fact, some of the properties we shall encounter have already been under focus: the combinatorial characterization exhibits surprising analogies with the class of House-Hole-Domino free graphs, see [13, 14], a sort of generalization of graphs admitting a perfect elimination ordering. These graphs arise as the result of looking for wider notions of ordering for graphs that still ensure nice computational properties. On the other hand, the algebraic characterization recalls the well known fact that graphs of fixed arbitrary tree-width may be constructed by means of an algebra of pushouts and relabelings [15]. The algebra of legal collapses suggests that, for entanglement, it might be possible 
to develop an analogous generic algebraic framework. It also points to standard graph theoretic ideas, such as $n$-connectiveness, as the proper tools by which to analyze entanglement.

Clearly, a work that still need to be carried out is to look for some useful characterization of directed graphs of entanglement at most $k$. At present, characterizations are known only for $k \leq 1$ [1, Proposition 3]. We believe that the results presented here suggest useful directions to achieve this goal. In particular, a suggestive path is to generalize the algebra of molecules and legal collapses to an undirected setting. This path might be a feasible one considering that many scientists have recently developed ideas and methods to lift some algebraic framework from an undirected to a directed setting. W.r.t. the algebra of entanglement, a source of ideas might be the recent development of directed homotopy theory from concurrency [16].

Acknowledgement. We would like to thank the anonymous referees for their useful comments, and for suggesting how to obtain the algorithm presented in Section 6 out of the algebraic framework introduced in Section 3.

\section{Entanglement Games}

The entanglement of a finite digraph $G$, denoted $\mathcal{E}(G)$, was defined in [1] by means of some games $\mathcal{E}(G, k), k=0, \ldots,\left|V_{G}\right|$. The game $\mathcal{E}(G, k)$ is played on the graph $G$ by Thief against Cops, a team of $k$ cops. The rules are as follows. Initially all the cops are placed outside the graph, Thief selects and occupies an initial vertex of $G$. After Thief's move, Cops may do nothing, may place a cop from outside the graph onto the vertex currently occupied by Thief, may move a cop already on the graph to the current vertex. In turn Thief must choose an edge outgoing from the current vertex whose target is not already occupied by some cop and move there. If no such edge exists, then Thief is caught and Cops win. Thief wins if he is never caught. The entanglement of $G$ is the least $k \in N$ such that $k$ cops have a strategy to catch the thief on $G$. It will be useful to formalize these notions.

Definition 1. The entanglement game $\mathcal{E}(G, k)$ of a digraph $G$ is defined by:

- Its positions are of the form $(v, C, P)$, where $v \in V_{G}, C \subseteq V_{G}$ and $|C| \leq k$, $P \in\{$ Cops, Thief $\}$.

- Initially Thief chooses $v_{0} \in V$ and moves to $\left(v_{0}, \emptyset\right.$, Cops).

- Cops can move from ( $v, C, C o p s)$ to $\left(v, C^{\prime}\right.$, Thief) where $C^{\prime}$ can be

1. C : Cops skip,

2. $C \cup\{v\}$ : Cops add a new Cop on the current position,

3. $(C \backslash\{x\}) \cup\{v\}$ : Cops move a placed Cop to the current position.

- Thief can move from ( $v, C$, Thief $)$ to $\left(v^{\prime}, C, C\right.$ ops $)$ if $\left(v, v^{\prime}\right) \in E_{G}$ and $v^{\prime} \notin C$.

Every finite play is a win for Cops, and every infinite play is a win for Thief. We let

$$
\mathcal{E}(G)=\min \{k \mid \text { Cops have a winning strategy in } \mathcal{E}(G, k)\}
$$


It is not difficult to argue that there exist polynomial time algorithms that, for fixed $k \geq 0$ decide on input $G$ whether $\mathcal{E}(G) \leq k$. Such an algorithm constructs the game $\mathcal{E}(G, k)$ whose size is polynomial in $\left|V_{G}\right|$ and $\left|E_{G}\right|$, since $k$ is fixed. Since the game $\mathcal{E}(G, k)$ is clopen, i.e. it is a parity game of depth 1 , it is well known [17] that such game can be solved in linear time w.r.t. the size of the graph underlying $\mathcal{E}(G, k)$.

In [1] the authors proved that $\mathcal{E}(G)=0$ if and only if it is $G$ is acyclic, and that $\mathcal{E}(G) \leq 1$ if and only if each strongly connected component of $G$ has a vertex whose removal makes the component acyclic. Using these results it was argued that deciding whether a graph has entanglement at most 1 is a problem in NLOGSPACE.

While wondering for a characterization of graphs of entanglement at most 2, we observed that such a question has a clear answer for undirected graphs.To deal with this kind of graphs, we recall that an undirected edge $\{u, v\}$ is just a pair $(u, v),(v, u)$ of directed edges. We can use the results of [1] to give characterizations of undirected graphs of entanglement at most 1 . To this goal, for $n \geq 0$ define the $n$-star of center $x_{0}$, noted $\varsigma_{x_{0}}^{n}$, to be the undirected graph $(V, E)$ where $V=\left\{x_{0}, a_{1}, \ldots, a_{n}\right\}$ and $E=\left\{\left\{x_{0}, a_{1}\right\}, \ldots,\left\{x_{0}, a_{n}\right\}\right\}$. More generally, say that a graph is a star if it is isomorphic to some $\varsigma_{x_{0}}^{n}$. Then we can easily deduce:

Proposition 2. If $G$ is an undirected graph, then $\mathcal{E}(G)=0$ if and only if $E_{G}=$ $\emptyset$, and $\mathcal{E}(G) \leq 1$ if and only if $G$ is a disjoint union of stars.

To end this section we state a Lemma that later will be used often. We remark that its scope does not restrict to undirected graphs.

Lemma 3. If $H$ is a subgraph of $G$ then $\mathcal{E}(H) \leq \mathcal{E}(G)$.

As a matter of fact, Thief can choose an initial vertex from $H$ and then he can restrict his moves to edges of $H$. In this way he can simulate a winning strategy from $\mathcal{E}(H, k)$ to a winning strategy in $\mathcal{E}(G, k)$.

\section{Molecules, Collapses, and the Class $\zeta_{2}$}

In this section we introduce a class of graphs and prove that the graphs in this class have entanglement at most 2. It will be the goal of the next sections to prove that these are all the graphs of entanglement at most 2 .

Definition 4. A molecule $\theta_{a, b}^{\varepsilon, n}$, where $\varepsilon \in\{0,1\}$ and $n \geq 0$, is the undirected graph $(V, E)$ with $V=\left\{a, b, c_{1}, \ldots, c_{n}\right\}$ and

$$
E= \begin{cases}\left.\left\{a, c_{1}\right\}, \ldots,\left\{a, c_{n}\right\},\left\{b, c_{1}\right\}, \ldots,\left\{b, c_{n}\right\}\right\}, & \varepsilon=0, \\ \left\{\{a, b\},\left\{a, c_{1}\right\}, \ldots,\left\{a, c_{n}\right\},\left\{b, c_{1}\right\}, \ldots,\left\{b, c_{n}\right\}\right\}, & \varepsilon=1 .\end{cases}
$$

The glue points of a molecule $\theta_{a, b}^{\varepsilon, n}$ are $a, b$. Its dead points are $c_{1}, \ldots, c_{n}$.

It is not difficult to prove that molecules have entanglement at most 2. 
Definition 5. Let $G_{1}$ and $G_{2}$ be two undirected graphs with $V_{G_{1}} \cap V_{G_{2}}=\emptyset$, let $a_{1} \in V_{G_{1}}$ and $a_{2} \in V_{G_{2}}$. The collapse of $G_{1}$ and $G_{2}$ on vertices $a_{1}$ and $a_{2}$, denoted $G_{1} \bigoplus_{a_{1}, a_{2}}^{z} G_{2}$, is the graph $G$ defined as follows:

$$
\begin{aligned}
V_{G}= & \left(V_{G_{1}} \backslash\left\{a_{1}\right\}\right) \cup\left(V_{G_{2}} \backslash\left\{a_{2}\right\}\right) \cup\{z\}, \text { where } z \notin V_{G_{1}} \cup V_{G_{2}}, \\
E_{G}= & \left\{\left\{x_{1}, y_{1}\right\} \in E_{G_{1}} \mid a_{1} \notin\left\{x_{1}, y_{1}\right\}\right\} \cup\left\{\left\{x_{2}, y_{2}\right\} \in E_{G_{2}} \mid a_{2} \notin\left\{x_{2}, y_{2}\right\}\right\} \\
& \cup\left\{\{x, z\} \mid\left\{x, a_{1}\right\} \in E_{G_{1}} \text { or }\left\{x, a_{2}\right\} \in E_{G_{2}}\right\} .
\end{aligned}
$$

We remark that $\bigoplus$ is a coproduct in the category of pointed undirected graphs and, for this reason, this operation is commutative and associative up to isomorphism. The graph $\eta$, whose set of vertices is a singleton, is a neutral element. As we have observed, a molecule is an undirected graph coming with a distinguished set of vertices, its glue points. Let us call a pair $(G, G l)$ with $G l \subseteq V_{G}$ a glue graph. For glue graphs we can define what it means that a collapse is legal.

Definition 6. If $G_{1}, G_{2}$ are glue graphs, then we say that $G_{1} \bigoplus_{a, b}^{z} G_{2}$ is a legal collapse if $a \in G l_{G_{1}}$ and $b \in G l_{G_{2}}$. We shall then use the notation $G_{1} \bar{\bigoplus}_{a, b}^{z} G_{2}$ and define

$$
G l_{G_{1}} \bar{\oplus}_{a, b}^{z} G_{2}=\left(G l_{G_{1}} \backslash\{a\}\right) \cup\left(G l_{G_{2}} \backslash\{b\}\right) \cup\{z\},
$$

so that $G_{1} \bar{\oplus}_{a, b}^{z} G_{2}$ is a glue graph.

Observe that the graph $\eta$ can be made into a unit for the legal collapse by letting $G l_{\eta}=V_{\eta}$. Even if the operation $\bar{\oplus}$ is well defined only after the choice of the two glue points that are going to be collapsed, it should be clear what it means that a family of glue graphs is closed under legal collapses.

Definition 7. We let $\zeta_{2}$ be the least class of glue graphs containing the molecules, the unit $\eta$, and closed under legal collapses and graph isomorphisms.

We need to make precise some notation and terminology. Firstly we shall abuse of notation and write

$$
G=H \bar{\bigoplus}_{v} K
$$

to mean that there exist subgraphs $H, K$ of $G$ such that $v \in G l_{G} \cap V_{H} \cap V_{K}$ and $G$ is isomorphic to the legal collapse $H \bar{\bigoplus}_{v, v}^{z} K$. Notice that if $H$ and $K$ are distinct from $\eta$, then $v$ is an articulation point of $G$. Second, we shall say that a graph $G$ belongs to $\zeta_{2}$ to mean that there exists a subset $G l \subseteq V_{G}$ such that the glue graph $(G, G l)$ belongs to $\zeta_{2}$. We can now state the main result of this section.

Proposition 8. If $G$ belongs to the class $\zeta_{2}$, then $\mathcal{E}(G) \leq 2$.

Proof. Observe that, given a molecule $\theta_{a, b}^{\varepsilon, n}$ occurring in an algebraic expression for $G$, we can rearrange the summands of the algebraic expression to write

$$
G=L \bar{\bigoplus}_{a} \theta_{a, b}^{\varepsilon, n} \bar{\bigoplus}_{b} R
$$


where $L, R \in \zeta_{2}$. A Cops winning strategy in the game $\mathcal{E}(G, 2)$ is summarized as follows. If Thief occupies some vertex of the molecule $\theta_{a, b}^{\varepsilon, n}$, Cops will place its two cops on $a$ and $b$, in some order. By doing that, Cops will force Thief to move (i) on the left component $L$, in which case Cops can reuse the cop on $b$ on $L$, (ii) on the molecule $\theta_{a, b}^{\varepsilon, n}$, in which case Thief will be caught in a dead point of the molecule, (iii) on the right component $R$, in which case Cops can reuse the cop on $a$ on $R$.

Cops can recursively use the same strategy in $\mathcal{E}(L, 2)$ and $\mathcal{E}(R, 2)$. The recursion terminates as soon as in the expression (1) for $G$ we have $L=R=\eta$.

The reader will have noticed similarities between the strategy proposed here and the strategy needed in [1] to argue that undirected trees have entanglement at most 2. As a matter of fact, graphs in $\zeta_{2}$ have an underlying tree structure. For a glue graph $G$, define the derived graph $\partial G$ as follows: its vertices are the glue points of $G$, and $\{a, b\} \in E_{\partial G}$ if either $\{a, b\} \in E_{G}$ or there exists $x \in V_{G} \backslash G l_{G}$ such that $\{a, x\},\{x, b\} \in E_{G}$. The following Proposition is not difficult to prove.

Proposition 9. A glue graph $G$ is in $\zeta_{2}$ if and only if $\partial G$ is a forest, and each $x \in V_{G} \backslash G l_{G}$ has exactly two neighbors, which moreover are glue points.

\section{Combinatorial Properties}

The goal of this section is to setup the tools for the characterization Theorem 16 . We deduce some combinatorial properties of undirected graphs of entanglement at most 2. To this goal, let us say that a simple cycle is long it its length is strictly greater than 4 , and say otherwise that it is short. Also, let us call a simple cycle of length 3 (resp. 4) a triangle (resp. square).

Proposition 10. An undirected graph $G$ such that $\mathcal{E}(G) \leq 2$ satisfies the following conditions:

- a simple Cycle of $G$ is Short,

- a triangle of $G$ has at least one vertex of degree 2 ,

- a square of $G$ cannot have two adjacent vertices

of degree strictly greater than 2. (No-AC)

Condition (No-3C) forbids as subgraphs of $G$ the graphs arising from the scheme on the left of figure 1. These are made up of a triangle and 3 distinct Collapses, with vertices $x, y, z$ that might not be distinct. Condition (No-AC) forbids the scheme on the right of figure 1, made up of a square and two Adjacent Collapses, with vertices $x, y$ that might not be distinct. Let us remark that graphs satisfying (CS), (No-3C), and (No-AC) are House-Hole-Domino free, in the sense of [14]. With respect to $H D D$-free graphs, the requirement is here stronger since for example long cycles are forbidden as subgraphs, not just as induced subgraphs. We shall see with Theorem 16 that these properties completely characterize 

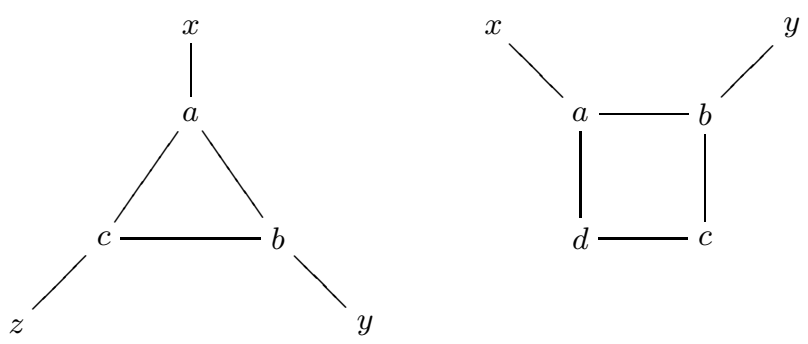

Fig. 1. The graphs $3 C$ and $A C$

the class of undirected graphs of entanglement at most 2. Proposition 10 is an immediate consequence of Lemma 3 and of the following Lemmas 11, 12, 13 .

Let $P_{0}$ be the empty graph and, for $n \geq 1$, let $P_{n}$ be the path with $n$ vertices and $n-1$ edges: $V_{P_{n}}=\{0, \ldots, n-1\}$ and $\{i, j\} \in E_{P_{n}}$ iff $|i-j|=1$. For $n \geq 3$, let $C_{n}$ be the cycle with $n$ vertices and edges: $V_{C_{n}}=\{0, \ldots, n-1\}$ and $\{i, j\} \in E_{C_{n}}$ iff $|i-j| \equiv 1 \bmod n$.

Lemma 11. If $n \geq 5$ then $\mathcal{E}\left(C_{n}\right) \geq 3$.

Proof. To describe a winning strategy for Thief in the game $\mathcal{E}\left(C_{n}, 2\right)$ consider that the removal of one or two vertices from $C_{n}$ transforms such graph into a disjoint union $P_{i}+P_{j}$ with $i+j \geq n-2 \geq 3$ : notice in particular that $i \geq 2$ or $j \geq 2$. In a position of the form (v,C,Thief) with $v \in C$, Thief moves to a component $P_{i}$ with $i \geq 2$. From a position of the form $(v, C$, Thief) with $v \notin C$, $v$ in some component $P_{i}$, and $i \geq 2$, Thief moves to some other vertex in the same component. This strategy can be iterated infinitely often, showing that Thief will never be caught.

Lemma 12. Let $3 C$ be a graph on the left of figure 1 . We have $\mathcal{E}(3 C) \geq 3$.

Proof. A winning strategy for Thief in the game $\mathcal{E}(3 C, 2)$ is as follows. By moving on $a, b, c$, Thief can force Cops to put two cops there, say for example on $a$ and $b$. Thief can then escape to $c$ and iterate moves on the edge $\{c, z\}$ to force Cops to move one cop on one end of this edge. From a position of the form $(c, C$, Thief $)$ with $c \in C$, Thief moves to a free vertex among $a, b$. From a position of the form $(z, C$, Thief) with $c \notin C$ Thief moves to $c$ and forces again Cops to occupy two vertices among $a, b, c$. Up to a renaming of vertices, such a strategy can be iterated infinitely often, showing that Thief will never be caught.

Observe that the proof does not depend on $x, y, z$ being distinct.

Lemma 13. Let $A C$ be a graph on the right of figure 1 . We have $\mathcal{E}(A C) \geq 3$.

Proof. By moving on $a, b, c, d$, Thief can force Cops to put two cops either on $a, c$ or on $b, d$ : let us say $a, c$. Thief can then escape to $b$ and iterate moves on 
the edge $\{b, y\}$ to force Cops to move one cop on one end of this edge. From a position of the form $(b, C$, Thief $)$ with $b \in C$, Thief moves to a free vertex among $a, c$. From a position of the form $(y, C$, Thief $)$ with $b \notin C$ Thief moves to $b$ and forces again Cops to occupy either $a, c$ or $b, d$. Up to a renaming of vertices, such a strategy can be iterated infinitely often, showing that Thief will never be caught. Again, we observe that the strategy does not depend on $x, y$ being distinct.

We end this section by pointing out that $\mathcal{E}\left(C_{n}\right)=\mathcal{E}(3 C)=\mathcal{E}(A C)=3(n \geq 5)$.

\section{Characterization of Entanglement at Most 2}

In this section we accomplish the characterization of the class of undirected graphs of entanglement at most 2: we prove that this class coincides with $\zeta_{2}$.

The following Lemma is the key observation by which the induction works in the proof of Proposition 15. It is worth, before stating it, to recall the difference between $\bigoplus$, the collapse of two ordinary undirected graphs, and $\bar{\oplus}$, the legal collapse of two glue graphs.

Lemma 14. Let $G$ be an undirected graph satisfying (No-3C) and (No-AC). If $G=\theta_{v, b}^{\varepsilon, n} \bigoplus_{b} H$ and $H \in \zeta_{2}$, then there is a subset $G l^{\prime} \subseteq V_{G}$ such that $\left(H, G l^{\prime}\right)$ is a glue graph in $\zeta_{2}, b \in G l^{\prime}$, and moreover $G$ is the result of the legal collapse $G=\theta_{v, b}^{\varepsilon, n} \bar{\bigoplus}_{b}\left(H, G l^{\prime}\right)$. Consequently, $G \in \zeta_{2}$, with $v$ a glue point of $G$.

The proof of the Lemma doesn't present difficulties and therefore it omitted.

Proposition 15. If $G$ is an undirected graph satisfying (CS), (No-3C), and (No-AC), then $G \in \zeta_{2}$.

Proof. The proof is by induction on $\left|V_{G}\right|$. Clearly the Proposition holds if $\left|V_{G}\right|=$ 1 , in which case $G=\eta \in \zeta_{2}$. Let us suppose the Proposition holds for all graphs $H$ such that $\left|V_{H}\right|<\left|V_{G}\right|$.

If all the vertices in $G$ have degree less than or equal to 2 , then $G$ is a disjoint union of paths and cycles of length at most 4 . Clearly such a graph belongs to $\zeta_{2}$. Otherwise, let $v_{0}$ be a vertex such that $\operatorname{deg}_{G}\left(v_{0}\right) \geq 3$ and consider the connected components $G_{\ell}, \ell=1, \ldots, h$, of the graph $G \backslash\left\{v_{0}\right\}$. Let $G_{\ell}^{v_{0}}$ be the subgraph of $G$ induced by $V_{G_{\ell}} \cup\left\{v_{0}\right\}$. We shall show that this graph is of the form

$$
G_{\ell}^{v_{0}}=\theta_{v_{0}, v_{1}}^{\varepsilon, m} \bigoplus_{v_{1}} H
$$

for some $\varepsilon \in\{0,1\}, m \geq 0$, and a graph $H \in \zeta_{2}$.

Clearly, if $G_{\ell}$ is already a connected component of $G$, then $G_{\ell} \in \zeta_{2}$ by the inductive hypothesis. We can pick any $v_{1} \in V_{G_{\ell}}$ and argue that formula (2) holds with $m=\varepsilon=0, H=G_{\ell}$.

Otherwise, let $\mathcal{N}_{\ell}=\left\{a_{1}, \ldots, a_{n}\right\}, n \geq 1$, be the set of vertices of $G_{\ell}^{v_{0}}$ at distance 1 from $v_{0}$. We claim that either the subgraph of $G_{\ell}$ induced by $\mathcal{N}_{\ell}$, noted $\mathcal{N}_{G_{\ell}}$, is a star or there exists a unique $v_{1} \in G_{\ell}$ at distance 1 from $\mathcal{N}_{\ell}$, and 
moreover the subgraph of $G_{\ell}$ induced by $\mathcal{N}_{\ell} \cup\left\{v_{1}\right\}$ is a star. In both cases, a vertex of such a star which is not the center has degree 2 in $G$.

(i) If $E_{\mathcal{N}_{G_{\ell}}} \neq \emptyset$, then $\mathcal{N}_{G_{\ell}}$ is a star. Let us suppose that $\left\{a_{1}, a_{2}\right\} \in E_{G_{\ell}}$. Since $G_{\ell}$ is connected, if $a_{k} \in \mathcal{N}_{\ell} \backslash\left\{a_{1}, a_{2}\right\}$ then there exists a path from $a_{k}$ to both $a_{1}$ and $a_{2}$. Condition (CS) implies that either $\left\{a_{1}, a_{k}\right\} \in E_{G_{\ell}}$, or $\left\{a_{k}, a_{2}\right\} \in E_{G_{\ell}}$. If $x_{0} \in V_{G_{\ell}} \backslash\left\{a_{2}\right\}$ then there cannot be a simple path $a_{k} \ldots x_{0} \ldots a_{1}$ otherwise $v_{0} a_{k} \ldots x_{0} \ldots a_{1} a_{2} v_{0}$ is a long cycle. Therefore, a simple path from $a_{k}$ to $a_{1}$ is of the form $a_{k} a_{1}$ or $a_{k} a_{2} a_{1}$. By condition (No-3C) it is not the case that $\left\{a_{k}, a_{1}\right\},\left\{a_{k}, a_{2}\right\} \in E_{G_{\ell}}$, otherwise $\left\{v_{0}, a_{1}, a_{2}, a_{k}\right\}$ is a clique of cardinality 4 . Finally, if $\left\{a_{k}, a_{1}\right\} \in E_{G_{\ell}}$ and $a_{l} \in \mathcal{N}_{\ell} \backslash\left\{a_{1}, a_{2}, a_{k}\right\}$, then $\left\{a_{l}, a_{1}\right\} \in E_{G_{\ell}}$ as well, by condition (CS), otherwise $v_{0} a_{k} a_{1} a_{2} a_{l} v_{0}$ is a long cycle. Therefore, if $\left|\mathcal{N}_{\ell}\right|>2$, then $\mathcal{N}_{G_{\ell}}$ is a star with a prescribed center, which we can assume to be $a_{1}$. Since $\operatorname{deg}_{G}\left(v_{0}\right) \geq 3$, by condition (No-3C) only $a_{1}$ among vertices in $\mathcal{N}_{\ell}$ may have degree greater than 2. Otherwise $\left|\mathcal{N}_{\ell}\right|=2$ and again at most one among $a_{i}$, $i=1,2$, has $\operatorname{deg}_{G}\left(a_{i}\right)>2$. Again, we can assume that $\operatorname{deg}_{G}\left(a_{2}\right)=2$. We deduce that the subgraph of $G_{\ell}^{v_{0}}$ induced by $\left\{v_{0}\right\} \cup \mathcal{N}_{\ell}$ is of the form $\theta_{v_{0}, a_{1}}^{1, n-1}$.

(ii) If $E_{\mathcal{N}_{G_{\ell}}}=\emptyset$, then we distinguish two cases. If $\left|\mathcal{N}_{\ell}\right|=1$, then the subgraph of $G_{\ell}^{v_{0}}$ induced by $\left\{v_{0}\right\} \cup \mathcal{N}_{\ell}$ is $\theta_{v_{0}, a_{1}}^{1,0}$. Otherwise, if $\left|\mathcal{N}_{\ell}\right| \geq 2$, between any two distinct vertices in $\mathcal{N}_{\ell}$ there must exist a path in $G_{\ell}$, since $G_{\ell}$ is connected. By condition (CS), if $a_{i} \ldots x_{i, j} \ldots a_{j}$ is a simple path from $a_{i}$ to $a_{j}$ with $x_{i, j} \in$ $V_{G_{\ell}} \backslash \mathcal{N}_{\ell}$, then $\left\{a_{i}, x_{i, j}\right\},\left\{a_{j}, x_{i, j}\right\} \in E_{G_{\ell}}$. Also (CS) implies that, for fixed $i$, $x_{i, k}=x_{i, j}$ if $k \neq j$, otherwise $v_{0} a_{k} x_{i, k} a_{i} x_{i, j} a_{j} v_{0}$ is a long cycle. We can also assume that $x_{i, j}=x_{j, i}$, and therefore $x_{i, j}=x_{i, k}=x_{l, k}$ whenever $i \neq j$ and $l \neq k$. Thus we can write $x_{i, j}=v_{1}$ for a unique $v_{1}$ at distance 2 from $v_{0}$. Since $\left|\mathcal{N}_{\ell}\right| \geq 2$ and $\operatorname{deg}_{G}\left(v_{0}\right) \geq 3$, condition (No-AC) implies that $\operatorname{deg}_{G}\left(a_{i}\right)=2$ for $i=1, \ldots, n$. We have shown that in this case the subgraph of $G_{\ell}^{v_{0}}$ induced by $\mathcal{N}_{\ell} \cup\left\{v_{0}, v_{1}\right\}$ is a molecule $\theta_{v_{0}, v_{1}}^{0, n}$, with $n \geq 2$.

Until now we have shown that (2) holds with $H$ a graph of entanglement at most 2. Since for such a graph $\left|V_{H}\right|<\left|V_{G}\right|$, the induction hypothesis implies $H \in \zeta_{2}$. Lemma 14 in turn implies that $G_{\ell}^{v_{0}} \in \zeta_{2}$, with $v_{0}$ a glue point of $G_{\ell}^{v_{0}}$. Finally we can use

$$
G=G_{1}^{v_{0}} \bar{\bigoplus}_{v_{0}} G_{2}^{v_{0}} \bar{\bigoplus}_{v_{0}} \ldots \bar{\bigoplus}_{v_{0}} G_{h}^{v_{0}}
$$

to deduce that $G \in \zeta_{2}$.

We can now state our main achievement.

Theorem 16. For a finite undirected graph $G$, the following are equivalent:

1. G has entanglement at most 2 ,

2. $G$ satisfies conditions (CS), (No-3C), (No-AC),

3. $G$ belongs to the class $\zeta_{2}$.

As a matter of fact, we have shown in the previous section that 1 implies 2 , in this section that 2 implies 3 , and in section 3 that 3 implies 1 . 


\section{A Linear Time Algorithm}

In this section we present a linear time algorithm that decides whether an undirected graph $G$ has entanglement at most 2 . We would like to thank the anonymous referee for pointing to us the ideas and tools needed to transform the algebraic characterization of Section 3 into a linear time algorithm.

Let us recall that, for $G=(V, E)$ and $v \in V, v$ is an articulation point of $G$ iff there exist distinct $v_{0}, v_{1} \in V \backslash\{v\}$ such that every path from $v_{0}$ to $v_{1}$ visits $v$. Equivalently, $v$ is an articulation point iff the subgraph of $G$ induced by $V \backslash\{v\}$ is disconnected. The graph $G$ is biconnected if it does not contain articulation points. A subset of vertices $V^{\prime} \subseteq V$ is biconnected iff the subgraph induced by $V^{\prime}$ is biconnected. A biconnected component of $G$ is biconnected subset $C \subseteq V$ such that if $C \subseteq V^{\prime}$ and $V^{\prime}$ is biconnected then $C=V$. The superstructure of $G$ is the graph $F_{G}$ defined as follows. Its set of vertices is the disjoint union $V_{F_{G}}=\mathcal{A}(G) \uplus \mathcal{C}(G)$, where

$$
\begin{aligned}
& \mathcal{A}(G)=\{a \in V \mid a \text { is an articulation point of } \mathrm{G}\}, \\
& \mathcal{C}(G)=\{C \subseteq V \mid C \text { is a biconnected component of } G\},
\end{aligned}
$$

and its set of edges is of the form

$$
E_{F_{G}}=\{\{a, C\} \mid a \in \mathcal{A}(G), C \in \mathcal{C}(G), \text { and } a \in C\} .
$$

It is well known that $F_{G}$ is a forest and that Depth-First-Search techniques may be used to compute the superstructure $F_{G}$ in time $O(|V|+|E|)$, see $[18, \S 23-2]$. Observe also that this implies that $\sum_{C \in \mathcal{C}(G)}|C|=O(|V|+|E|)$. This relation that may also be derived considering that biconnecetd components do not share common edges, so that $\left|V_{F_{G}}\right|=O(|V|+|E|)$ and $\left|E_{F_{G}}\right|=O(|V|+|E|)$ since $F_{G}$ is a forest. We have therefore

$$
\begin{aligned}
\sum_{C \in \mathcal{C}(G)}|C| & =|V \backslash \mathcal{A}(G)|+\sum_{a \in \mathcal{A}(G)}|\{C \in \mathcal{C}(G) \mid a \in C\}| \\
& =|V \backslash \mathcal{A}(G)|+\left|E_{F_{G}}\right|=O(|V|+|E|) .
\end{aligned}
$$

The algorithm ENTANGLEMENT-TWO relies on the following considerations. If a graph $G$ belongs to the class $\zeta_{2}$, then it has an algebraic expression explaining how to construct it using molecules as building blocks and legal collapses as operations. We can assume that in this expression the molecule $\theta_{a, b}^{0,1}$ does not appear, since each such occurrence may be replaced by the collapse $\theta_{a, x}^{1,0} \bar{\bigoplus}_{x} \theta_{x, b}^{1,0}$. W.r.t. this normalized expression, if $G$ is connected then its articulation points are exactly those glue points $v$ of $G$ that appears in the algebraic expression as subscripts of some legal collapse $\bar{\bigoplus}_{v}$; the molecules are the biconnected components of $G$.

The algorithm computes the articulation points and the biconnected components of $G$ - that is, its superstructure - and afterwards it checks that each biconnected component together with its articulation points is a molecule. 


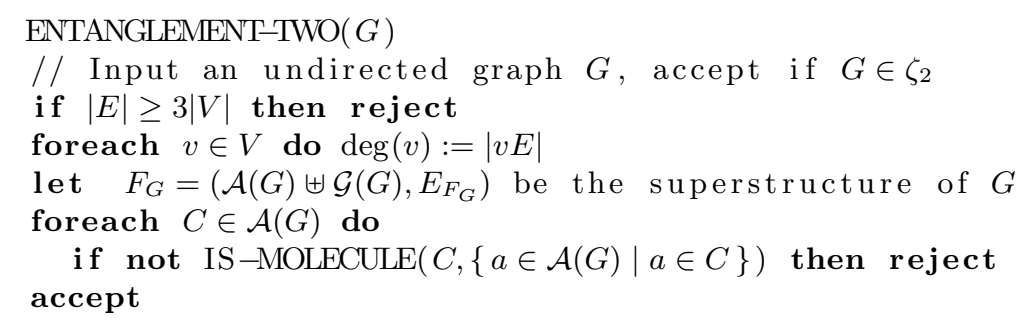

For a biconnected component together with a set of candidate glue points to be a molecule we need of course these candidates to be at most 2. Also, every vertex whose degree in $G$ is not 2 is a candidate glue point. Improving on these observations we arrive at the following characterization.

Lemma 17. Let $G=(V, E)$ be a biconnected graph and $D \subseteq V$ be such that $\{v \in V \mid \operatorname{deg}(v) \neq 2\} \subseteq D$. Then $G$ is isomorphic to a molecule $\theta_{a, b}^{\epsilon, n}$, with $D$ isomorphically sent to a subset of $\{a, b\}$, if and only if either (i) $|D|=2$ and $\{x, d\} \in E$ for each $x \in V \backslash D$ and $d \in D$ or (ii) $|D|<2$ and $|V| \in\{3,4\}$.

Therefore the recognition algorithm for a molecule is as follows.

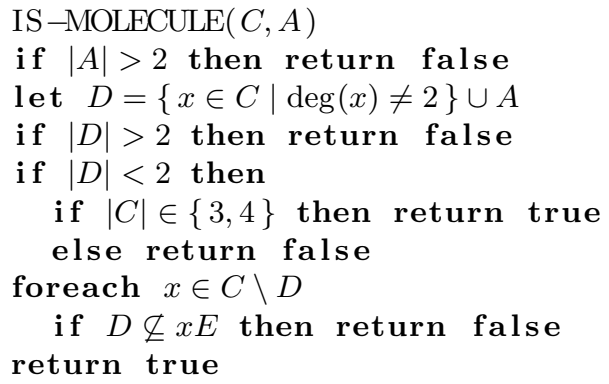

Let us now argue about time resources of this algorithm.

Fact. Algorithm ENTANGLEMENT-TWO(G) runs in time $O\left(\left|V_{G}\right|\right)$.

It is clear that the function IS-MOLECULE runs in time $O(|C|)$, so that the loop (lines 7-8) of ENTANGLEMENT-TWO runs in time $O\left(\sum_{C \in \mathcal{C}(G)}|C|\right)=$ $O(|V|+|E|)$. Therefore the algorithm requires time $O(|V|+|E|)$.

The following Lemma, whose proof depends on considering a tree with back edges arising from a Depth-First-Search on the graph, elucidates the role of the 3rd line of the algorithm.

Lemma 18. If a graph $(V, E)$ does not contain a simple cycle $C_{n}$ with $n \geq k$, then it has at most $(k-2)|V|-1$ undirected edges.

Line 3 ensures $\left|E_{G}\right|=O\left(\left|V_{G}\right|\right)$ and that the algorithm runs in time $O\left(\left|V_{G}\right|\right)$. 


\section{References}

1. Berwanger, D., Grädel, E.: Entanglement-a measure for the complexity of directed graphs with applications to logic and games. In: Logic for programming, artificial intelligence, and reasoning. Volume 3452 of Lecture Notes in Comput. Sci. Springer, Berlin (2005) 209-223

2. Berwanger, D.: Games and Logical Expressiveness. PhD thesis, RWTH Aachen (2005)

3. Seymour, P.D., Thomas, R.: Graph searching and a min-max theorem for treewidth. J. Combin. Theory Ser. B 58(1) (1993) 22-33

4. Gottlob, G., Leone, N., Scarcello, F.: Hypertree decompositions: A survey. In: MFCS '01: Proceedings of the 26th International Symposium on Mathematical Foundations of Computer Science, London, UK, Springer-Verlag (2001) 37-57

5. Johnson, T., Robertson, N., Seymour, P.D., Thomas, R.: Directed tree-width. J. Combin. Theory Ser. B 82(1) (2001) 138-154

6. Safari, M.A.: d-width: a more natural measure for directed tree width. In: Mathematical foundations of computer science 2005. Volume 3618 of Lecture Notes in Comput. Sci. Springer, Berlin (2005) 745-756

7. Berwanger, D., Dawar, A., Hunter, P., Kreutzer, S.: Dag-width and parity games. In Durand, B., Thomas, W., eds.: STACS. Volume 3884 of Lecture Notes in Computer Science., Springer (2006) 524-536

8. Berwanger, D., Grädel, E., Lenzi, G.: On the variable hierarchy of the modal mucalculus. In Bradfield, J., ed.: Computer Science Logic, CSL 2002. Volume 2471 of LNCS., Springer-Verlag (2002) 352-366

9. Berwanger, D., Lenzi, G.: The variable hierarchy of the $\mu$-calculus is strict. In: STACS 2005, Proceedings of the 22nd Symposium on Theoretical Aspects of Computer Science. Volume 3404 of LNCS., Springer-Verlag (2005) 97-109

10. Kozen, D.: Results on the propositional $\mu$-calculus. Theoret. Comput. Sci. 27(3) (1983) 333-354

11. Arnold, A., Niwiński, D.: Rudiments of $\mu$-calculus. Volume 146 of Studies in Logic and the Foundations of Mathematics. North-Holland Publishing Co., Amsterdam (2001)

12. Bloom, S.L., Ésik, Z.: Iteration theories. Springer-Verlag, Berlin (1993)

13. Jamison, B., Olariu, S.: On the semi-perfect elimination. Adv. in Appl. Math. 9(3) (1988) 364-376

14. Chepoi, V., Dragan, F.: Finding a central vertex in an HHD-free graph. Discrete Appl. Math. 131(1) (2003) 93-111

15. Courcelle, B.: Graph rewriting: an algebraic and logic approach. In: Handbook of theoretical computer science, Vol. B. Elsevier, Amsterdam (1990) 193-242

16. Goubault, E., Raußen, M.: Dihomotopy as a tool in state space analysis. In Rajsbaum, S., ed.: LATIN. Volume 2286 of Lecture Notes in Computer Science., Springer (2002) 16-37

17. Jurdzinski, M.: Small progress measures for solving parity games. In Reichel, H., Tison, S., eds.: STACS. Volume 1770 of Lecture Notes in Computer Science., Springer (2000) 290-301

18. Cormen, T.H., Leiserson, C.E., Rivest, R.L.: Introduction to Algorithms. first edn. The MIT Electrical Engineering and Computer Science Series. The MIT Press (1990) 